\title{
A Novel ex vivo Mouse Mesometrium Culture Model for Investigating Angiogenesis in Microvascular Networks
}

\author{
Ariana D. Suarez-Martinez ${ }^{\mathrm{a}, \mathrm{b}}$ Susanne Bierschenk ${ }^{\mathrm{c}}$ Katie Huang ${ }^{\mathrm{d}}$ \\ Dana Kaplan $^{b}$ Carolyn L. Bayer ${ }^{b}$ Stryder M. Meadows ${ }^{d}$ Markus Sperandio $^{c}$ \\ Walter L. Murfee ${ }^{a, b}$ \\ a J. Crayton Pruitt Family Department of Biomedical Engineering, University of Florida, Gainesville, FL, USA; \\ ${ }^{\mathrm{b}}$ Department of Biomedical Engineering, Tulane University, New Orleans, LA, USA; ${ }^{C}$ Walter-Brendel-Centre of \\ Experimental Medicine, Klinikum der Universität München, Ludwig-Maximilians-Universität, Munich, Germany; \\ ${ }^{\mathrm{d}}$ Department of Cell and Molecular Biology, Tulane University, New Orleans, LA, USA
}

\section{Keywords}

Microcirculation · Angiogenesis · Microvascular network ·

Capillary sprouting $\cdot$ Endothelial cell $\cdot$ Pericyte

\begin{abstract}
Background: The development of models that incorporate intact microvascular networks enables the investigation of multicellular dynamics during angiogenesis. Our laboratory introduced the rat mesentery culture model as such a tool, which would be enhanced with mouse tissue. Since mouse mesentery is avascular, an alternative is mouse mesometrium, the connective tissue of uterine horns. The study's objective was to demonstrate that mouse mesometrium contains microvascular networks that can be cultured to investigate multicellular dynamics during angiogenesis. Methods: Harvested mesometrium tissues from $\mathrm{C} 57 \mathrm{BI} / 6$ female mice were cultured in media with serum for up to 7 days. PECAM, NG2, aSMA, and LYVE-1 labeling identified endothelial cells, pericytes, smooth muscle cells, and lymphatic endothelial cells, respectively. Results: These cells comprised microvascular networks with arterioles, venules, and capillaries. Compared to day 0 , capillary sprouts per vascular length were increased
\end{abstract}

๑) 2018 S. Karger AG, Basel by 3 and 5 days in culture (day $0,0.08 \pm 0.01$; day $3,3.19 \pm$ 0.78 ; day $5,2.49 \pm 0.05$ sprouts $/ \mathrm{mm} ; p<0.05$ ). Time-lapse imaging of cultured tissues from FlkEGFP mice showcases the use of the model for lineage studies. The impact is supported by the identification of endothelial cell jumping from one sprout to another. Conclusion: These results introduce a novel culture model for investigating multicellular dynamics during angiogenesis in real-time ex vivo microvascular networks.

(c) 2018 S. Karger AG, Basel

\section{Introduction}

As microvascular remodeling is a common denominator for multiple pathologies such as tumor growth, myocardial ischemia, and diabetes [1], a need exists to understand the multicellular dynamics involved in angiogenesis, defined as the growth of new blood vessels from preexisting ones. This drive to understand angiogenesis has inspired the emergence and use of tissue-engineered, biomimetic ex vivo models, which enables views not possible in vivo for basic science discovery. However, the challenge to recapitulate the complexity of angiogenesis in intact mi-

\section{KARGER}

E-Mail karger@karger.com

www.karger.com/jvr
Dr. Walter Lee Murfee

J. Crayton Pruitt Family Department of Biomedical Engineering, University of Florida Biomedical Sciences Building, J284, 1275 Center Drive

Gainesville, FL 32611 (USA)

E-Mail wmurfee@bme.ufl.edu 
crovascular networks in a dish highlights a gap between in vitro and in vivo. In real tissue, capillary sprouting off existing vessels incorporates the dynamics of endothelial cells, smooth muscle cells, pericytes, and interstitial cells [2]. This complexity is further increased with interactions between the blood microvascular system and lymphatic or neural systems. Therefore, a critical question that addresses the biomimetic model challenge is: how can we incorporate the multicellular and multisystem complexity of angiogenesis in microvascular networks?

Common models such as two-dimensional [3], threedimensional [4], and microfluidic [5] approaches have built up complexity and proven to be extremely useful. However, each model is limited by the fact that they do not look and function like real networks. As an alternative to the bottom-up approaches, our laboratory recently introduced the rat mesentery culture model, in which the microvascular complexity of real tissues is maintained in culture. Advantages of the rat mesentery culture model are: (1) multiple cell types (i.e., endothelial cells, smooth muscle cells, pericytes, and macrophages) remain viable along the hierarchy of microvascular networks [6-8], (2) angiogenesis occurs preferentially along venules and capillaries versus arterioles similar to in vivo [6], (3) smooth muscle cells and pericytes remain functional $[6,9]$, and (4) the ability to simultaneously capture time-lapse images of angiogenesis and lymphangiogenesis [7, 10]. Leveraging these characteristics, the applicability of this model would be enhanced with the use of transgenic mouse tissue, yet unfortunately the same connective tissue in mouse is avascular [11].

A potential analog to the rat mesentery is the mouse mesometrium, which is the thin connective tissue of the uterine horns, yet it is unknown whether it contains a microvasculature. The objective of this study was to demonstrate that the mouse mesometrium contains intact microvascular networks and that angiogenesis can be induced in culture. Our results: (1) confirmed that mouse mesometrium has intact microvascular networks with multiple cell types, (2) demonstrated the ability to induce angiogenesis in culture, and (3) allowed for time-lapse lineage studies by using FlkEGFP mice. Our findings introduce the mouse mesometrium culture model as a novel tool for real-time ex vivo investigations of angiogenesis.

\section{Materials and Methods}

Mouse Mesometrium Tissue Harvesting and Culture Model

All animal experiments were approved by Tulane University's Institutional Animal and Care Use Committee. Adult, female
$\mathrm{C} 57 \mathrm{Bl} / 6$ mice were euthanized by asphyxiation in a $\mathrm{CO}_{2}$ chamber followed by cervical dislocation. The abdominal fur was shaved before sterilizing the abdomen with $70 \%$ isopropyl alcohol followed by 3 wipes with iodine. The abdominal skin and muscles were cut off with sterile scissors to expose the organs. Two forceps were utilized to move away all organs until the uterine horns were exposed. Each uterine horn was stretched to clearly expose the mesometrium, the connective tissue attaching the uterine horns to the back wall, and then pinned down to facilitate harvesting. Sterile saline warmed to $37^{\circ} \mathrm{C}$ was dripped on the tissue to prevent dehydration as needed. Using aseptic techniques, the mesometrium tissues were harvested with forceps and microscissors by pinching a side of the mesometrium and cutting around the uterine horn (Fig. 1a). Once removed, the tissues were immediately rinsed in sterile Dulbecco's phosphate-buffered saline (DPBS; Gibco), and then transferred to sterile minimum essential media (MEM; Gibco) and $1 \%$ penicillin-streptomycin (PenStrep; Gibco) warmed to $37^{\circ} \mathrm{C}$. The mesometrium tissues were transferred to a biosafety cabinet after harvesting. Using a 6-well culture plate, $4 \mathrm{~mL}$ of culture media was transferred into each well where a single tissue was placed. The culture media was comprised of sterile MEM and 1\% PenStrep. Tissues were then immediately placed inside an incubator set at standard cell culture conditions $\left(5 \% \mathrm{CO}_{2}, 37^{\circ} \mathrm{C}\right)$ for up to 7 days, where the media was changed every $24 \mathrm{~h}$.

\section{Immunohistochemistry}

Tissues were first spread on a microscope slide and then fixed in methanol at $-20{ }^{\circ} \mathrm{C}$ for $30 \mathrm{~min}$ before labeling. The following antibodies were used to label the tissues: 1:200 mouse platelet endothelial cell adhesion molecule (PECAM; CD31) with 1:500 streptavidin secondary (Strep-CY2), 1:200 CY3-conjugated $\alpha$-smooth muscle actin (aSMA), 1:100 rabbit neuron-glial antigen 2 (NG2) with 1:100 goat anti-rabbit secondary (GAR-CY3), and 1:100 rabbit lymphatic vessel endothelial hyaluronan receptor-1 (LYVE-1) with 1:100 GAR-CY3. Staining protocols were replicated as previously stated by Stapor et al. [6]. Briefly, all antibodies were diluted in antibody buffer solution which contained PBS $+0.1 \%$ saponin $+2 \%$ bovine serum albumin. All primary and secondary antibodies were incubated for $1 \mathrm{~h}$ at room temperature. After every antibody incubation, tissues were rinsed 3 times with cold PBS $+0.1 \%$ saponin for $10 \mathrm{~min}$.

\section{Stimulation of Angiogenesis}

To induce angiogenesis, tissues were cultured in sterile MEM supplemented with $20 \%$ fetal bovine serum (FBS; Gibco) or 400 $\mathrm{ng} / \mathrm{mL}$ of recombinant mouse vascular endothelial growth factor-164 (VEGF164; R\&D Systems). The tissues were placed in the following groups: (1) day 0 ( $n=8$ tissues from 4 mice), (2) day 3 with MEM $+20 \%$ FBS ( $n=8$ tissues from 4 mice), (3) day 3 with MEM ( $n=8$ tissues from 4 mice), (4) day 5 with MEM $+20 \%$ FBS ( $n=8$ tissues from 4 mice), (5) day 5 with MEM ( $n=8$ tissues from 4 mice), and (6) day 5 with MEM + VEGF ( $n=6$ tissues from 3 mice). Tissues from the day 0 group were fixed and labeled immediately after being excised.

\section{Quantification of Angiogenesis}

Using the Java-based NIH ImageJ v.1.51 image processing software, capillary sprouts were quantified for the entire tissue. Capillary sprouts were defined as blind-ended PECAM-positive endothelial cell segments protruding from existing microvessels. The 


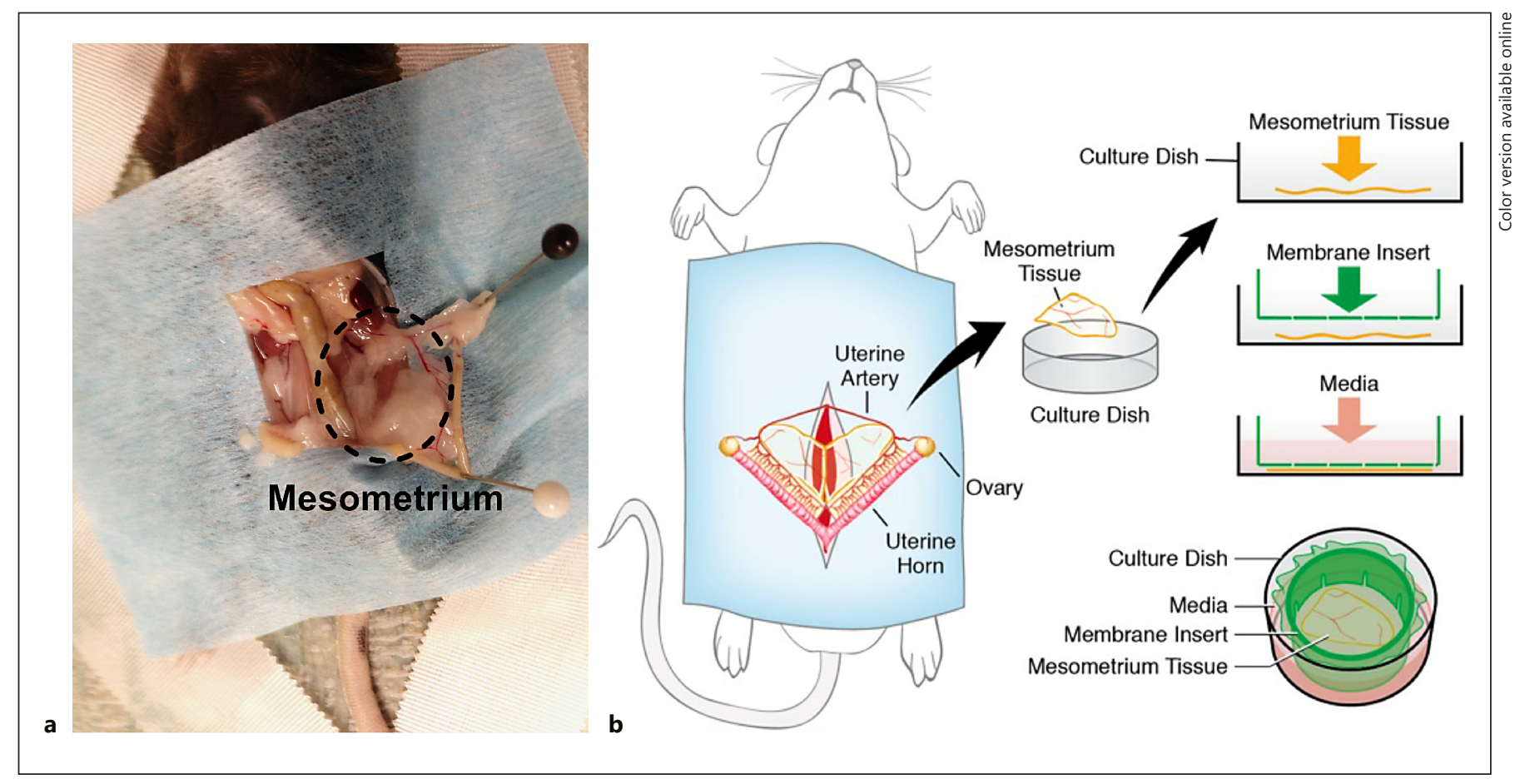

Fig. 1. Mouse mesometrium culture model. Image showing a mouse with the mesometrium tissue exposed (dashed oval; a) and a schematic demonstrating the harvested tissue for time-lapse imaging during culture (b).

data were normalized per total vessel length. To differentiate between arterioles and venules, the diameter of the blood vessel and the morphology of endothelial cells were examined since arterioles are known to have smaller diameters and more elongated endothelial cells due to their higher shear stress compared to paired venules $[12,13]$. All arterioles and venules that measured larger than $7 \mu \mathrm{m}$ in diameter were analyzed.

\section{Microscopy}

Images were taken with a $4 \times($ dry, $\mathrm{NA}=0.1), 10 \times($ dry, $\mathrm{NA}=$ 0.3 ), and $20 \times$ (oil, $\mathrm{NA}=0.8$, or air, $\mathrm{NA}=0.75$ ) objectives from an inverted microscope (Olympus IX71) paired with a Photometrics CoolSNAP EZ camera. Whole tissue images with PECAM labeling were acquired with a $4 \times$ or $10 \times$ objective. Time-lapse images were captured using the $10 \times($ dry, $\mathrm{NA}=0.3)$ objective.

\section{Time-Lapse Imaging of Endothelial Cell Dynamics}

Additional mesometrium tissues from adult, female FlkEGFP mice with a CD1 background were harvested using the same aseptic techniques. These transgenic mice were graciously provided by Dr. Meadows' lab from the Department of Cell and Molecular Biology at Tulane University. In the biosafety cabinet, tissues were spread on a 1- $\mu \mathrm{m}$ polycarbonate filter fitted to a cell-crown insert (Sigma-Aldrich) and then inverted into a well from a 6-well culture plate with $4 \mathrm{~mL}$ of media supplemented with $20 \%$ FBS (Fig. 1b). The culture plate was immediately placed in the incubator, and media was changed every $24 \mathrm{~h}$. Images of the same venule were acquired with the $10 \times$ objective at least once every $24 \mathrm{~h}$ for 7 days.
For day 0 and 1, an image was captured every $24 \mathrm{~h}$; for day 2 and 3 , images were captured every $4 \mathrm{~h}$, and for days $4-7$, images were captured every $6 \mathrm{~h}$.

\section{Statistical Analysis}

Data are presented as the mean \pm standard error of the mean (SEM). Angiogenesis data with serum were compared across experimental groups with 2-way analysis of variance (ANOVA) followed by Tukey multiple comparisons tests to notify differences between media and serum and between time periods. Angiogenesis data with VEGF was compared using an unpaired 2-tailed Student $t$ test. A $p$ value $<0.05$ was considered statistically significant. Statistical analysis was executed using GraphPad Prism version 7 software.

\section{Results}

\section{Mouse Mesometrium Tissue Contains Intact \\ Microvascular Networks}

PECAM-positive labeling of endothelial cells along the hierarchy of branched microvascular networks was present in the mesometrium, which is the thin connective tissue of the uterine horns in female mice (Fig. 2b). In contrast, tissues harvested from the mouse mesentery were 
Fig. 2. Comparison of the microvasculature between mesentery and mesometrium tissue from C57Bl/6 female mice. PECAM labeling identified endothelial cells along intact microvascular networks. a Image showing the avascular region of the mouse mesentery tissue. b Image showing the vascular region of the mouse mesometrium tissue. The lines denote the border between the connective tissue and the adipose tissue $\left.{ }^{*}\right)$. A, arteriole; V, venule; C, capillary. Scale bars, $100 \mu \mathrm{m}$.

Fig. 3. Microvascular characterization of the mesometrium tissue. Mouse mesometrium contains PECAM-positive endothelial cells comprising intact microvascular networks with distinguishable arterioles, venules, and capillaries (a), along with supporting cells including aSMA-positive smooth muscle cells (b), and NG2-positive pericytes (c). $\mathbf{d}$ In addition to blood vessels, the mesometrium also contains LYVE1-positive lymphatic vessels. A, arteriole; $\mathrm{V}$, venule; L, lymphatic; C, capillary. Arrows show wrapping pericytes. Arrowheads show interstitial LYVE-1-positive cells aligning themselves to capillaries. Asterisks $\left(^{*}\right)$ identify adipocytes. Scale bars, $100 \mu \mathrm{m}$.
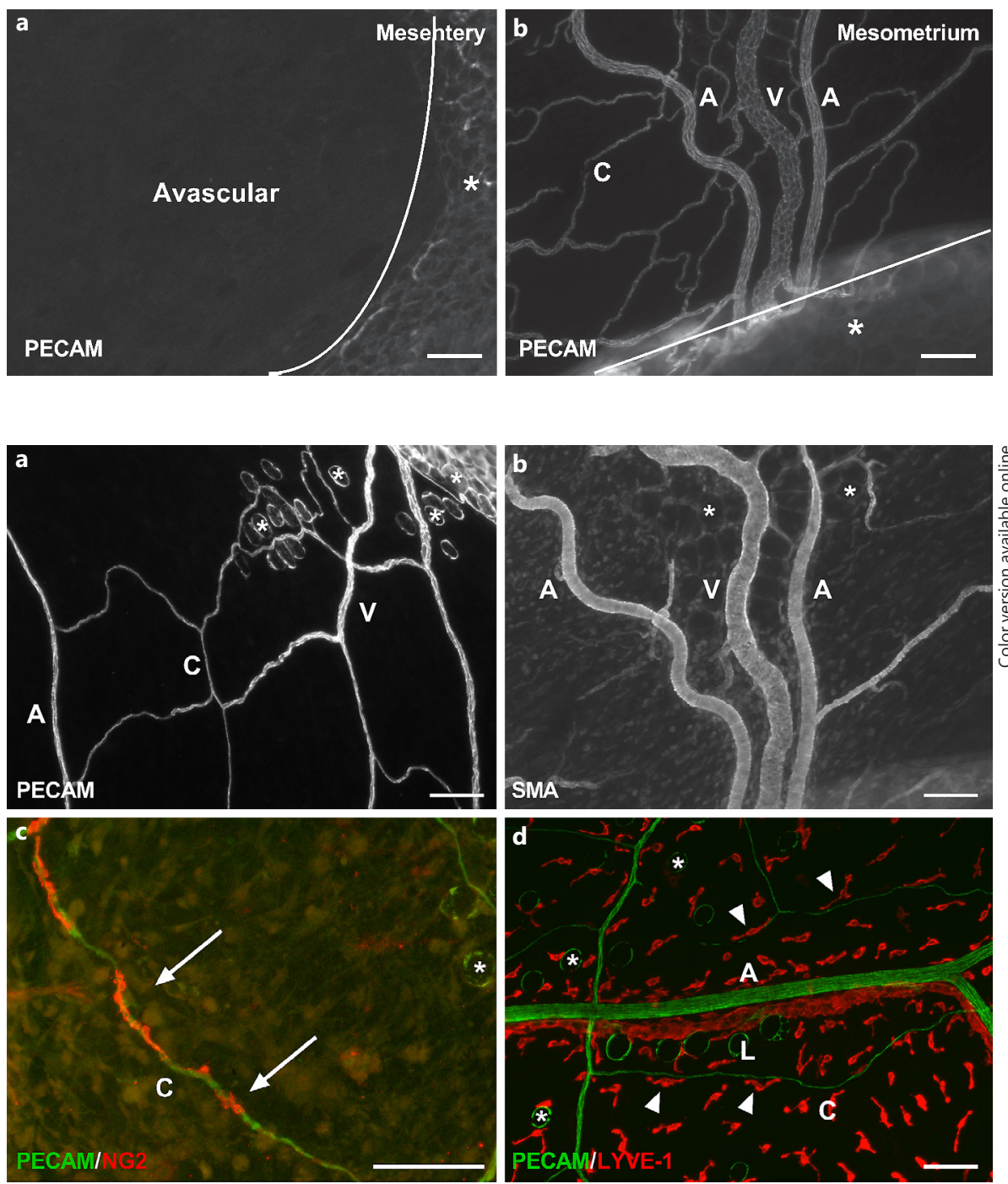

avascular as expected and displayed no evidence of PECAM-positive labeled networks (Fig. 2a). Intact networks in the mesometrium were commonly characterized by branching arterioles, venules, and capillaries (Fig. 2b, 3a). Vessel types were identified based on endothelial cell morphology and relative vessel diameters [12, 13]. Importantly, aSMA-positive smooth muscle cells and NG2-positive pericytes were present in their expected locations and displayed typical wrapping morphologies (Fig. 3b, c). Mesometrium tissues also demonstrated the presence of LYVE-1-positive lymphatics (Fig. 3d). Lymphatics were distinguished by the endothelial cell morphology, intensity of PECAM, and LYVE-1-positive labeling.

\section{Angiogenesis Can Be Stimulated in Cultured Mouse Mesometrium Tissue}

Angiogenesis, defined as capillary sprouts, was observed in mouse mesometrium tissues cultured in MEM supplemented with 20\% FBS for 3 and 5 days (Fig. 4a-c). The number of PECAM-positive endothelial cell sprouts per total vascular length significantly increased for the groups supplemented with FBS for 3 days (MEM $+20 \%$ FBS, $3.19 \pm 0.78$ sprouts/mm; MEM, $0.60 \pm 0.18$ sprouts/ $\mathrm{mm} ; p<0.0001)$ and 5 days (MEM + 20\% FBS, $2.49 \pm 0.05$ sprouts/mm; MEM, $0.80 \pm 0.13$ sprouts $/ \mathrm{mm} ; p=0.0033$ ) in culture when compared to the MEM control group (Fig. 4d-f). During culture, tissue viability was confirmed via Live/Dead assay (online suppl. Fig. 1; see www. karger.com/doi/10.1159/000489102 for all online suppl. 

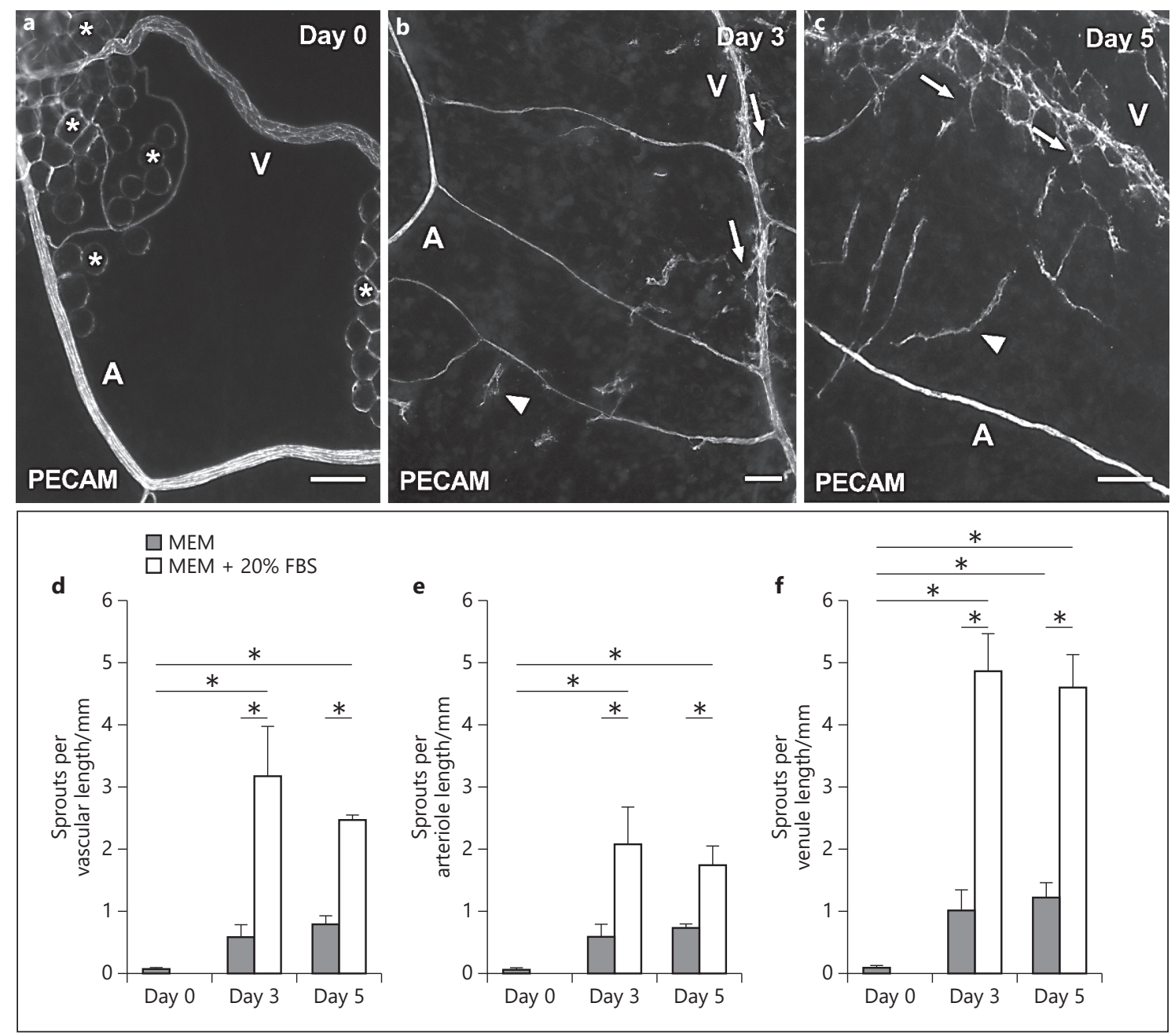

Fig. 4. Angiogenesis is stimulated during culture in intact microvascular networks from mouse mesometrium tissues. Tissues were cultured with MEM supplemented with $20 \%$ FBS for 3 days (b) and 5 days (c) and compared to day 0 tissues (a). Asterisks $\left({ }^{*}\right)$ identify adipocytes. The angiogenic response was quantified by counting the number of capillary sprouts (arrows), defined as blind-ended, PECAM-positive sprouts coming off microvessels, and normaliz-

material). MEM supplemented with VEGF was also sufficient to induce an increase in the number of PECAMpositive endothelial cell sprouts per total vascular length after 5 days in culture, as seen in Figure 5 (MEM, $0.80 \pm$ 0.13 sprouts $/ \mathrm{mm}$; MEM + VEGF, $2.35 \pm 0.57$ sprouts $/ \mathrm{mm}$; $p=0.0031)$. Capillary sprouts preferentially originated off venules compared to arterioles in all the angiogenic groups, which is consistent with in vivo observations [14]. While the capillary sprout density was comparable at day 3 and day 5, the length of capillary sprouts qualitatively appeared to be longer in day 5 tissues (data not shown). ing it by the total length of vessels. The total number of sprouts per total vascular length (d), number of sprouts from arterioles per arteriole length (e), and number of sprouts from venules per venule length (f) are shown. The arrowheads indicate vascular islands. $\mathrm{A}$, arteriole; $\mathrm{V}$, venule. Black bars represent MEM alone, and white bars represent MEM supplemented with 20\% FBS. Data are presented as the mean $+\mathrm{SEM} ;{ }^{*} p<0.05$. Scale bars, $100 \mu \mathrm{m}$. 

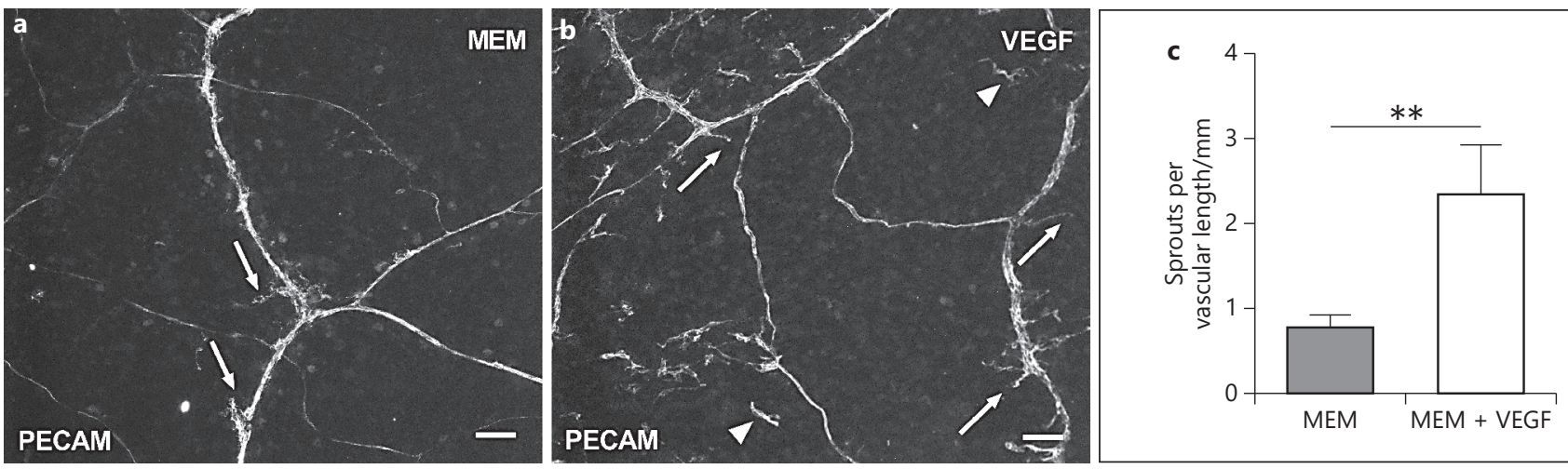

Fig. 5. VEGF media supplementation stimulates angiogenesis. The mouse mesometrium tissues were cultured with MEM (a) or with MEM supplemented with VEGF (b) for 5 days. This resulted in capillary sprouts (arrows) and the presence of vascular islands (ar-

rowheads), which are defined as disconnected endothelial segments. The angiogenic response was quantified by counting the total number of sprouts per total vascular length $(\mathbf{c})$. Data are presented as the mean + SEM; ${ }^{* *} p<0.005$.

ing angiogenesis in culture, NG2-positive cells were present along venules (Fig. 6d-i). Along capillary sprouts, pericytes were typically NG2 positive (Fig. $6 \mathrm{~d}-\mathrm{i}$ ) and aSMA negative (Fig. 6a-c).

Time-Lapse Imaging Reveals Novel Endothelial Cell

Dynamics during Capillary Sprouting

Time-lapse imaging of cultured mesometrium tissues harvested from FlkEGFP mice enabled the tracking of endothelial cell dynamics during capillary sprouting (Fig. 7). Images recorded at least every $24 \mathrm{~h}$ revealed "endothelial cell jumping," defined by an endothelial cell disconnecting from a capillary sprout and subsequently connecting to a neighboring capillary sprout (Fig. 8; online suppl. movie).

\section{Discussion}

The main contributions of this study are the identification of microvascular networks in female mouse mesometrium connective tissue and the establishment of the mouse mesometrium culture model as an ex vivo tool for investigating cellular dynamics involved in angiogenesis. Recently, our laboratory introduced the rat mesentery culture model for real-time investigation of pericyte-endothelial cell interactions during angiogenesis in intact networks [6-9]. The maintenance of physiological complexity associated with a real network highlights the advantages of the top-down tissue culture approach versus more common bottom-up tissue engineering approaches, such as two- and three-dimensional cell culture $[3,4]$ and microfluidic models [5]. However, the application of our mesentery culture approach for cell lineage or mechanistic studies is limited by the use of rat tissue, the lack of varied transgenic rat strains, and the absence of microvasculature in mouse mesentery. Other analogous connective tissues include the omentum and the mouse mesometrium. Since our observations suggest the omentum connective tissue is also void of microvascular networks (data not shown), the current study focused on the mesometrium. To our knowledge, these results are the first to show that mesometrium tissue harvested from adult, female mice contains intact multicellular microvascular networks with arterioles, venules, and capillaries. Furthermore, the ability to increase capillary sprouting in culture via serum or growth factor media supplementation supports its use as a novel ex vivo model for discovering cell dynamics involved in angiogenesis.

The use of transgenic mouse strains and the application of the mouse mesometrium culture model as a new dynamic view of angiogenesis within intact networks is highlighted by tracking FlkEGFP-positive endothelial cell sprouting off a venule. Our time-lapse observations suggest a phenomenon that we term "endothelial cell jumping." Endothelial cells can disconnect from one sprout segment and then reconnect to a neighboring sprout segment. While the functional contribution of "endothelial cell jumping" remains unknown, this finding is consistent with observations of capillary sprouting in mosaic embryoid body cell culture [16]. This suggests an alternative endothelial cell dynamic in an adult tissue that challenges common sprout paradigms. Interestingly, we also observed examples of dramatic increases in the number 

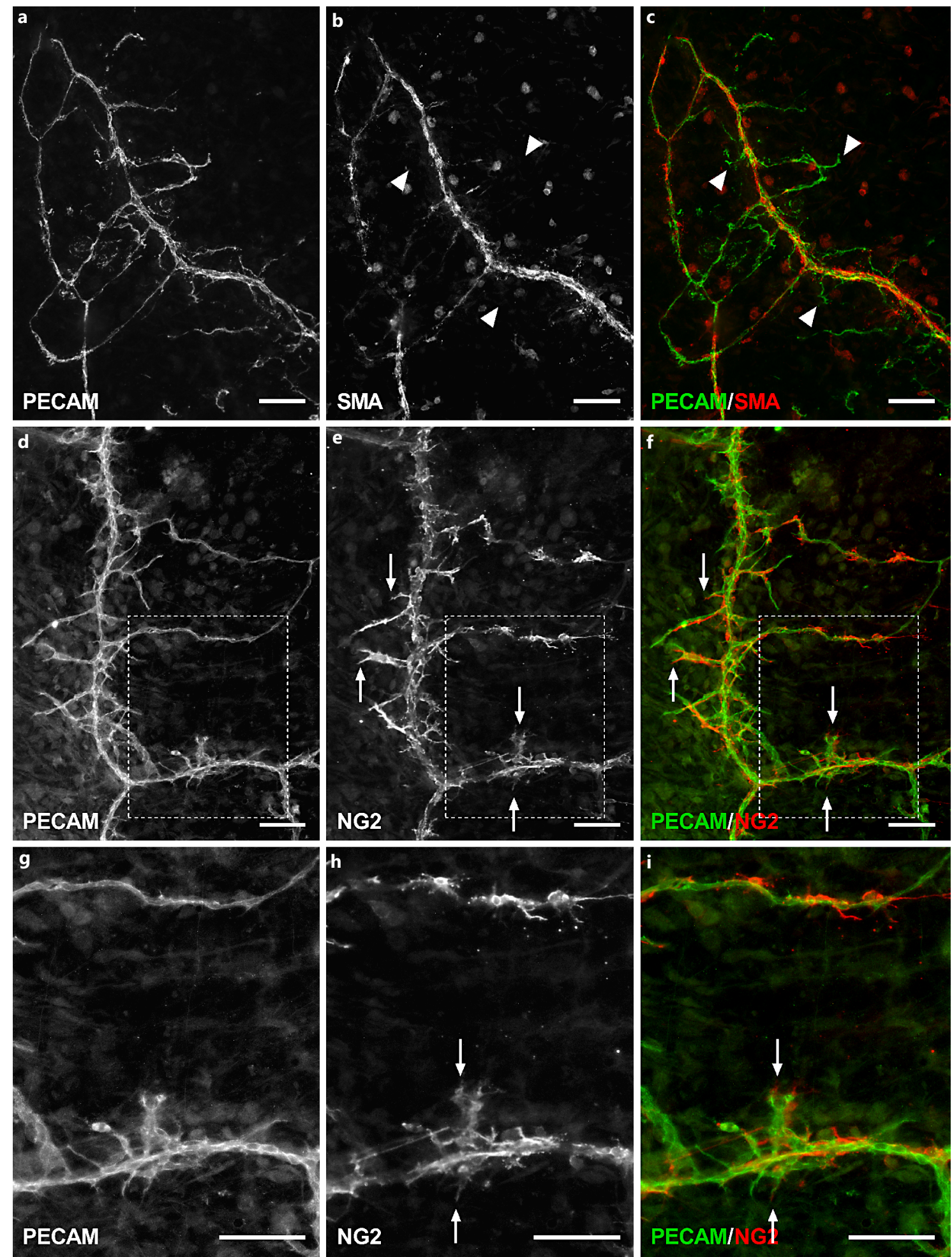

Fig. 6. Representative images of aSMA and NG2 perivascular cell labeling during angiogenesis stimulated with 20\% FBS for 3 days. Mesometrium tissues labeled with PECAM and aSMA antibodies (a-c) show only PECAM-positive leading sprouts (arrowheads).

In contrast, mesometrium tissues labeled with PECAM and NG2 antibodies (d-i) show NG2-positive cells leading sprouts (arrows). Images $\mathbf{g}$-i are higher magnifications of the tissue regions indicated by the dashed boxes. Scale bars, $100 \mu \mathrm{m}$. 

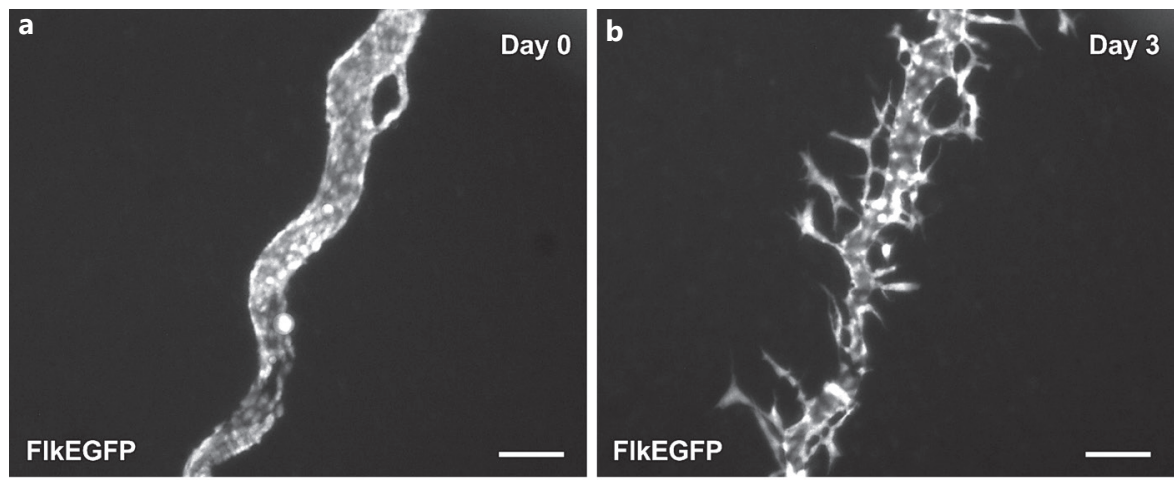

Fig. 7. Time-lapse images of a venule in the mesometrium tissue from a FlkEGFP mouse during culture. Images of a venule at the same location were acquired for days 0 (a), 3 (b), 5 (c), and 7 (d). Scale bars, 100 $\mu \mathrm{m}$.
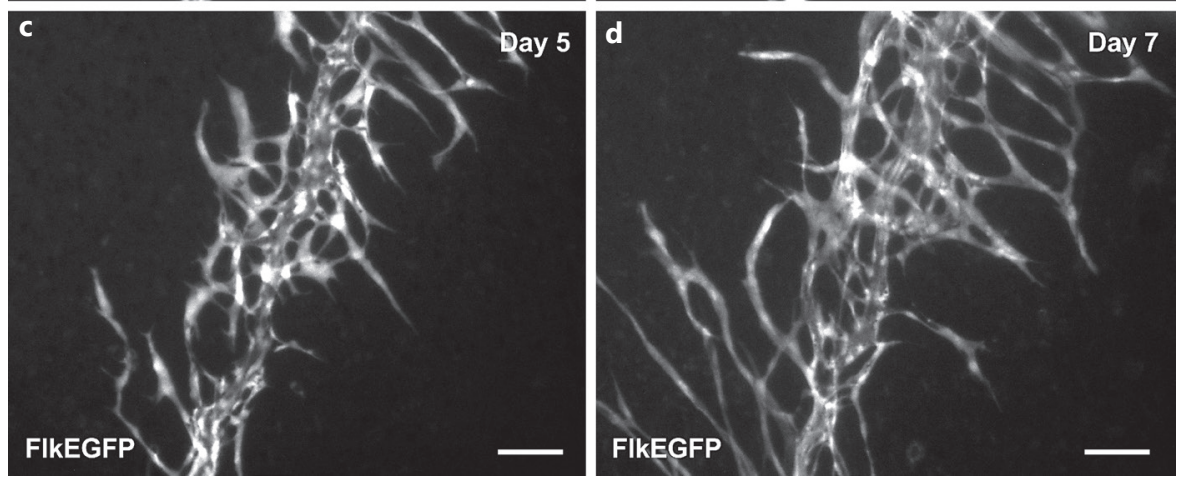

of vascular islands during angiogenesis in cultured mesometrium tissues (data not shown). We have previously discovered that vascular islands, defined as disconnected endothelial segments, have the ability to reconnect with growing microvascular networks $[6,17,18]$. The new discovery of "endothelial cell jumping" suggests that disconnected endothelial segments might originate via migration from existing vessels.

Similar to rat mesentery connective tissue, mouse mesometrium is thin and can be easily harvested. The tissue can be whole-mounted and provides an essential two-dimensional view down to the single cell level of an intact three-dimensional microvascular network. Since the mesometrium tissue is self-contained, it does not require embedding into a three-dimensional matrix for culturing. Compared to more common models, an advantage of the mesometrium culture model is that capillary sprouting can be quantified at specific locations within a network. While the model currently lacks perfusion, the preferential sprouting off venules and capillaries versus arterioles is physiologically relevant and supports in vivo studies [14]. The physiological relevance is also supported by the presence of smooth muscle cells and pericytes. These two cell types display similar phenotypes as in vivo, supported by: (1) the presence of NG2-positive and
aSMA-negative cells leading PECAM-positive capillary sprouts in serum-stimulated tissues, (2) the lack of NG2 labeling along venules in unstimulated tissues, and (3) the presence of NG2-positive perivascular cells along venules during angiogenesis $[15,19-22]$.

An obvious constraint of the mouse mesometrium tissue is that it limits studies to female mice and does not allow for the evaluation of sex differences. Another limitation is the unknown tissue-specific aspects compared to the omentum or the mesentery. Interestingly in the mouse, the mesentery and omentum are avascular and future studies are needed to identify why vessels are normally present in the mesometrium. Additional studies are also warranted to determine how microvascular remodeling dynamics in the mesometrium are the same or different compared to other vascularized tissues like the rat mesentery. While our results demonstrate that the mesometrium has similar cell types comprising an intact microvascular network comparable to the rat mesentery and that angiogenesis can be stimulated in culture, the specific mechanisms for growth in the mesometrium remain unknown.

In the current study, angiogenesis was stimulated by media supplemented with $20 \%$ serum for up to 7 days. Since an objective of this study was to stimulate angiogen- 

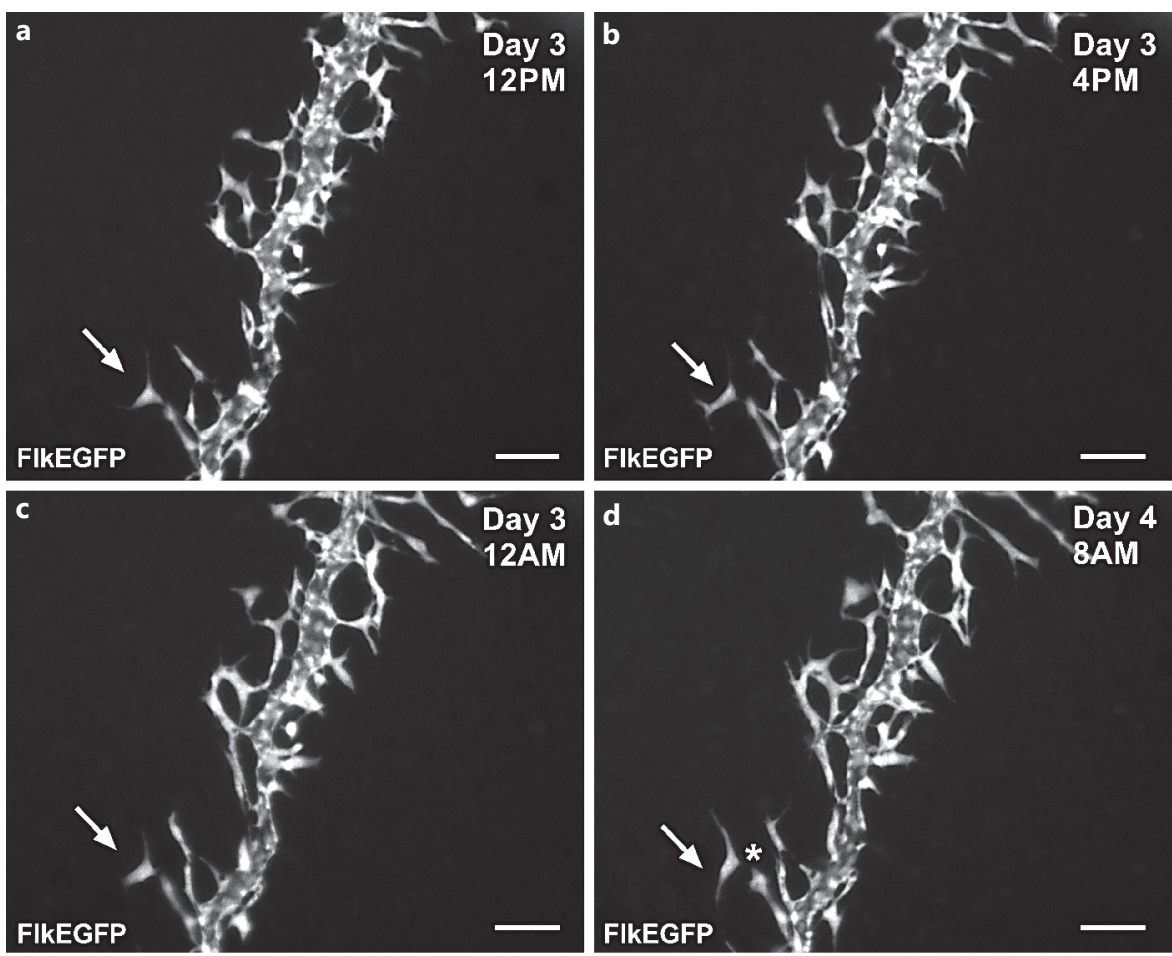

Fig. 8. Time-lapse images of a venule from a FlkEGFP mouse showing "endothelial cell jumping" during 3 and 4 days in culture. Jumping is defined as the disconnection of an endothelial cell sprout that subsequently attaches itself to a neighboring sprout. Arrows highlight the endothelial cell that jumped from one sprout to another, and the asterisk $(*)$ indicates the original host sprout for the jumping endothelial cell. Scale bars, $100 \mu \mathrm{m}$.
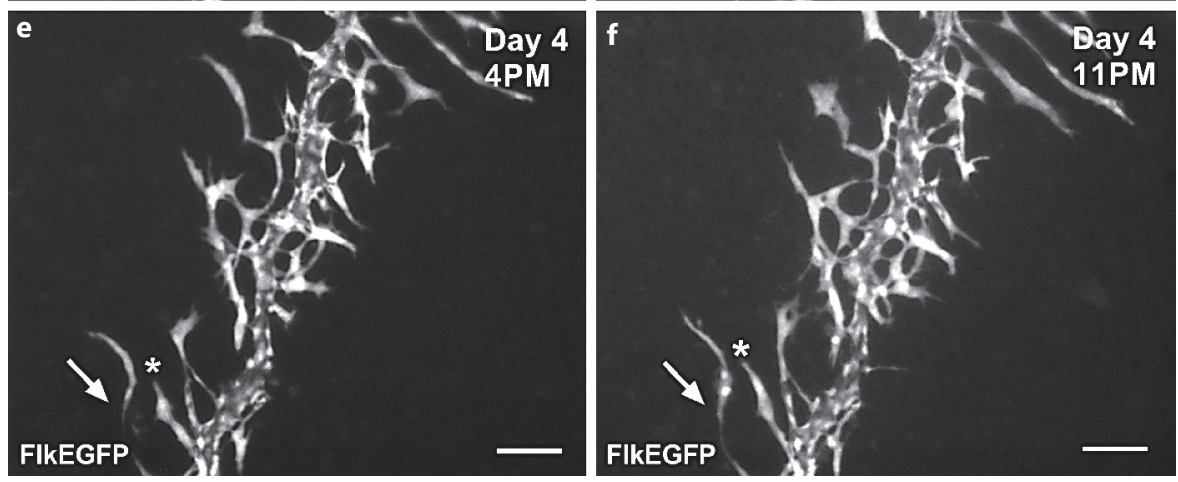

esis in the mouse mesometrium, serum was selected because it has been shown to cause a robust increase in capillary sprouting in the rat mesentery culture model $[6,7]$. Our results also suggest that VEGF can cause sprouting, yet future experiments will be needed to confirm whether pericyte and endothelial cell phenotypic dynamics are similar to media-supplemented tissues. Both day 3 and day 5 serum-stimulated tissues displayed an increase in capillary sprouting. Furthermore, qualitative observations suggest that day 5 sprouts were longer than day 3 sprouts, suggesting that the angiogenic effect progresses over time. Longer time point experiments will also be needed to determine the duration for which tissues can be kept in culture and the subsequent stages of vessel growth. Previous work in our laboratory has established that rat mesentery tissues remain viable for at least 7 days [6]. We have also observed viable cells and networks with high endothelial segment density in rat mesentery tissues cultured out to 14 days (data not shown). Based on our previous experiences with culturing rat mesentery tissue and the observation of remodeling in the cultured FlkEGFP mesometrium tissues at 7 days, we speculate that mouse mesometrium tissues can be cultured past 7 days.

In addition to containing blood microvascular networks, a subset of mouse mesometrium tissues also contained examples of LYVE-1-positive lymphatic networks. Lymphatic vessels, identified by LYVE-1-positive expression and morphology, displayed similar characteristics to lymphatic vessels in rat mesentery tissues [8, 23-25]. Interestingly, LYVE-1-positive cells were also observed in 
the interstitial space with a subpopulation aligning themselves with the microvessels and some even hugging vessels (Fig. 3d). These LYVE-1-positive interstitial cells appear morphologically similar to mural lymphatic endothelial cells which were recently discovered by Bower et al. [26] in zebrafish larvae. The LYVE-1 labeling in the mesometrium coupled with the ability to use specific transgenic mice motivate the future use of the mouse mesometrium culture model for novel cell tracking experiments. These studies will be aimed at identifying the origin and fate of the novel lymphatic interstitial cells and the coordination between angiogenesis and lymphangiogenesis.

In conclusion, our results introduce the mouse mesometrium as an alternative connective tissue to the rat mesentery for studying angiogenesis. As the rat mesentery has proven valuable for discovering cell phenotypic dynamics during microvascular network growth $[15,25$, 27-29], we envision that the mouse mesometrium will offer a similar impactful view for researchers. Since the mouse mesentery is void of microvascular networks, the mouse mesometrium offers a connective tissue alternative for ex vivo culture and enables new types of real-time cell fate studies.

\section{Acknowledgements}

Dr. Murfee would like to thank the Microcirculatory Society for supporting his visit to Dr. Markus Sperandio's laboratory in the Walter Brendel Centre of Experimental Medicine (WBex) at Ludwig-Maximilians-Universität München. Dr. Murfee especially thanks Dr. Ulrich Pohl, chairman of WBex, for making the visit possible and Dr. Sperandio and the members of his laboratory for sharing their research environment.

\section{Disclosure Statement}

The authors do not have any conflicts of interests.

\section{Funding Sources}

The research reported in this publication was supported by the National Institute of Aging under Award No. R01AG049821 (W.L.M) and Tulane University start-up funds (S.M.M).

\section{References}

1 Carmeliet P: Angiogenesis in life, disease and medicine. Nature 2005;438:932-936.

2 Adams RH, Alitalo K: Molecular regulation of angiogenesis and lymphangiogenesis. Nat Rev Mol Cell Biol 2007;8:464-478.

3 Goodwin AM: In vitro assays of angiogenesis for assessment of angiogenic and anti-angiogenic agents. Microvasc Res 2007;74:172183.

4 DelNero P, Lane M, Verbridge SS, Kwee B, Kermani P, Hempstead B, et al: 3D culture broadly regulates tumor cell hypoxia response and angiogenesis via pro-inflammatory pathways. Biomaterials 2015;55:110-118.

5 Song JW, Munn LL: Fluid forces control endothelial sprouting. Proc Natl Acad Sci USA 2011;108:15342-15347.

-6 Stapor PC, Azimi MS, Ahsan T, Murfee WL: An angiogenesis model for investigating multicellular interactions across intact microvascular networks. AJP Heart Circ Physiol 2013; 304:H235-H245.

7 Azimi MS, Myers L, Lacey M, Stewart SA, Shi Q, Katakam PV, et al: An ex vivo model for anti-angiogenic drug testing on intact microvascular networks. PLoS One 2015; 10: e0119227.
8 Sweat RS, Sloas DC, Murfee WL: VEGF-C induces lymphangiogenesis and angiogenesis in the rat mesentery culture model. Microcirculation 2014;21:532-540.

-9 Motherwell JM, Azimi MS, Spicer K, Alves NG, Hodges NA, Breslin JW, et al: Evaluation of arteriolar smooth muscle cell function in an ex vivo microvascular network model. Sci Rep 2017;7:2195.

10 Sweat RS, Sloas DC, Stewart SA, Czarny-Ratajczak M, Baddoo M, Eastwood JR, et al: Aging is associated with impaired angiogenesis, but normal microvascular network structure, in the rat mesentery. Am J Physiol Heart Circ Physiol 2017;312:H275-H284.

11 Norrby K: In vivo models of angiogenesis. J Cell Mol Med 2006;10:588-612.

12 Murfee W, Skalak T, Peirce S: Differential arterial/venous expression of NG2 proteoglycan in perivascular cells along microvessels: identifying a venule-specific phenotype. Microcirculation 2005; 12:151-160.

13 Yang M, Aragon M, Murfee WL: Angiogenesis in mesenteric microvascular networks from spontaneously hypertensive versus normotensive rats: angiogenesis in hypertensive rat mesentery. Microcirculation 2011;18: 574-582.
14 Rhodin J, Fujita H: Capillary growth in the mesentery of normal young rats - intravital video and electron microscope analyses. J Submicrosc Cytol Pathol 1989;21:1-34.

15 Murfee WL, Rehorn MR, Peirce SM, Skalak TC: Perivascular cells along venules upregulate NG2 expression during microvascular remodeling. Microcirculation 2006;13:261273.

16 Jakobsson L, Franco CA, Bentley K, Collins RT, Ponsioen B, Aspalter IM, et al: Endothelial cells dynamically compete for the tip cell position during angiogenic sprouting. Nat Cell Biol 2010;12:943-953.

-17 Kelly-Goss MR, Winterer ER, Stapor PC, Yang M, Sweat RS, Stallcup WB, et al: Cell proliferation along vascular islands during microvascular network growth. BMC Physiol 2012;12:7.

18 Kelly-Goss MR, Sweat RS, Azimi MS, Murfee WL: Vascular islands during microvascular regression and regrowth in adult networks. Front Physiol 2013;4:108.

19 Kelly-Goss MR, Sweat RS, Stapor PC, Peirce SM, Murfee WL: Targeting pericytes for angiogenic therapies. Microcirculation 2014;21: 345-357. 
20 Nehls V, Drenckhahn D: Heterogeneity of microvascular pericytes for smooth muscle type alpha-actin. J Cell Biol 1991;113:147154.

21 Ponce AM, Price RJ: Angiogenic stimulus determines the positioning of pericytes within capillary sprouts in vivo. Microvasc Res 2003; 65:45-48.

22 Stapor PC, Sweat RS, Dashti DC, Betancourt AM, Murfee WL: Pericyte dynamics during angiogenesis: new insights from new identities. J Vasc Res 2014;51:163-174.

23 Benest AV, Harper SJ, Herttuala SY, Alitalo K, Bates DO: VEGF-C induced angiogenesis preferentially occurs at a distance from lymphangiogenesis. Cardiovasc Res 2008;78: 315-323.
24 Robichaux JL, Tanno E, Rappleye JW, Ceballos M, Stallcup WB, Schmid-Schönbein GW, et al: Lymphatic/blood endothelial cell connections at the capillary level in adult rat mesentery. Anat Rec Adv Integr Anat Evol Biol 2010;293:1629-1638.

25 Sweat RS, Stapor PC, Murfee WL: Relationships between lymphangiogenesis and angiogenesis during inflammation in rat mesentery microvascular networks. Lymphat Res Biol 2012;10:198-207.
Bower NI, Koltowska K, Pichol-Thievend C, Virshup I, Paterson S, Lagendijk AK, et al: Mural lymphatic endothelial cells regulate meningeal angiogenesis in the zebrafish. Nat Neurosci 2017;20:774-783.

-27 Anderson CR, Hastings NE, Blackman BR, Price RJ: Capillary sprout endothelial cells exhibit a CD $36^{\text {low }}$ phenotype. Am J Pathol 2008; 173:1220-1228.

28 Stapor PC, Murfee WL: Identification of class III $\beta$-tubulin as a marker of angiogenic perivascular cells. Microvasc Res 2012;83:257262.

29 Benest AV, Bates DO: Measurement of angiogenic phenotype by use of two-dimensional mesenteric angiogenesis assay. Angiogenesis Protoc 2009;467:251-270. 